\title{
Plant Tissue Culture Technology: Sustainable Option for Mining High Value Pharmaceutical Compounds
}

\author{
M.K. Tripathi, Nishi Mishra, Sushma Tiwari*, Chitralekha Shyam, \\ Sonali Singh and Ashok Ahuja \\ Department of Plant Molecular Biology \& Biotechnology, College of Agriculture, \\ RVSKVV, Gwalior 474002, MP, India \\ *Corresponding author
}

\section{Keywords}

Tissue culture, Pharmaceutical compounds, Secondary metabolism

Article Info

Accepted:

10 January 2019

Available Online:

10 February 2019

\section{A B S T R A C T}

Production of secondary metabolites from plant tissue culture has emerged as a promising and feasible option attracting the attention of scientists worldwide. Plant cell, tissue and organ cultures offer an attractive alternative for homogeneous, controlled production of secondary metabolites, throughout the year, especially when we take commercial demand into account. They not only facilitate the de novo synthesis of novel compounds, but also are able to produce compounds sometimes even in higher amounts than the intact plants. Many biotechnological strategies have been experimented for enhanced production of secondary metabolites from medicinal plants. Some of these include screening of high yielding cell lines, media modification, precursor feeding, elicitation, large scale cultivation in bioreactor system, hairy root culture, plant cell immobilization, biotransformation and many others. Some of the recent developments such as metabolic engineering of whole plants and plant cell cultures have been established as effective tools to increase metabolites yield. The use of genetic engineering tools and elucidation of pathways for secondary metabolism has provided the basis for the production of commercially acceptable levels of product. In view of commercial importance of the secondary metabolites as high value pharmaceutical compounds in recent years resulted in a immersive interest, in secondary metabolism, and particularly in the possibility to alter the production of bioactive plant metabolites by utilizing biotechnological tools. The plant cell culture technology provides sustainable option for production of plant pharmaceuticals and could be used for the large-scale production of metabolites.

\section{Introduction}

Plant cell culture systems are potential renewable source of valuable medicinal compounds, flavors, fragrances, and colorants. Due to commercial importance of the secondary metabolites it has resulted in an interest in secondary metabolism. Production of bioactive plant metabolites by means of cell culture technology has gained interest and number of plants has been investigated in vitro in recent years to produce compounds of medicinal value. This technology provide continuous, reliable source of plant 
pharmaceuticals and could be used for the large-scale cultivation of plant cells in bioreactors and through downstreaming processes these metabolites can be extracted (Balandrin and Klocke, 1988). In addition to its importance in the discovery of new medicines, plant cell culture technology plays an even more significant role in solving world hunger by developing agricultural crops that provide both higher yield and more resistance to pathogens and adverse environmental and climatic conditions. This paper reviews some of the developments for the production of some of the bioactive secondary metabolites from medicinal plants.

Medicinal plants are the important source of life saving drugs for the majority of the world's population. Biologically active secondary metabolite compounds extracted from plants are used as food additives, pigments, dyes, insecticides, cosmetics and perfumes and fine chemicals (Ahuja, 1988). These compounds commonly referred as secondary metabolites. Number of plant species that are used as medicinal herbs have been scientifically evaluated for their possible medical applications. Due to wild crafting owing to developmental activities natural stands of many medicinal plants are disappearing fast and together with environmental and geopolitical instabilities; it is increasingly difficult to meet the demand. As such to face such challenges industries, as well as scientists have to look for the possibilities of alternative resources for the production of plant pharmaceuticals utilizing plant cell cultures. Advances in biotechnology in recent years particularly methods for culturing plant cell cultures, has provided good strategies for the commercial processing of plant cell cultures even rare plants and the chemicals they provide. As such there has been considerable interest in plant cell cultures as a potential alternative to traditional agriculture for the industrial production of secondary metabolites (Rao and Ravishankar, 2000). Plant cell culture technologies were introduced at the end of $1960^{\mathrm{s}}$ as a possible tool for both studying and producing plant secondary metabolites.

Different strategies using cell cultures systems have been extensively studied with the objective of improving the production of bioactive secondary metabolites. Cell culture systems could be used for the large scale culturing of plant cells from which secondary metabolites can be extracted. The advantage of this method is that it can ultimately provide a continuous, reliable source of natural products. The major advantages of cell cultures includes (i) synthesis of bioactive secondary metabolites in controlled environment, independently from climatic and soil conditions; (ii) negative biological influences that affect secondary metabolites production in the nature are eliminated (microorganisms and insects); (iii) it is possible to select cultivars with higher production of secondary metabolites; (iv) with automatization of cell growth control and metabolic processes regulation cost price can decrease and production increase. The objectives of many industries are to develop plant cell culture techniques to the stage where they yield secondary products more cheaply than extracting either the whole plant grown under natural conditions or synthesizing the product. Although the production of pharmaceuticals using plant cell cultures have been highlighted, other uses have also been suggested as new route for synthesis, for products from plants difficult to grow, or in short supply, as a source of novel chemicals and as biotransformation systems (Ramawat et al., 1999; Oksman-Caldentey and Inze, 2004). Recent research results indicate that plant cell suspension cells can be used for recombinant protein production under controlled conditions (Verpoorte and Memelink, 2002). Some of the successful 
cases where callus and cell suspension cultures have been described for production of bioactive secondary metabolites obtained by authors are described.

\section{Secondary metabolites production by plant cell cultures}

For plant cell culture techniques to become economically viable, it is important to develop methods that would allow for consistent generation of high yields of products from cultured cells. Careful selection of productive cells and cultural conditions resulted in accumulation of several products in higher levels in cultured cells. In order to obtain yields in higher concentrations for commercial exploitation, efforts have focused on the stimulation of biosynthetic activities of cultured cells using various methods (Rao and Ravishankar, 2000).Culture productivity is critical to the practical application of plant cell culture technology to production of plantspecific bioactive metabolites. Until now, various strategies have been developed to improve the production of secondary metabolites using plant cell cultures. The tissue culture cells typically accumulate large amounts of secondary compounds only under specific conditions. That means maximization of the production and accumulation of secondary metabolites by plant tissue cultured cells requires (i) manipulating the parameters of the environment and medium, (ii) selecting high yielding cell clones, (iii) precursor feeding and (iv) elicitation.

Plant cell cultures are mostly a heterogeneous system in which individual plant cells are different physiologically. High yielding lines selection and screening of plant cell cultures have been described by many researchers (Rao and Ravishankar, 2000). Exogenous supply of biosynthetic precursors to culture medium is another important strategy to increase the yield of desired products. This approach is useful when the precursors are inexpensive. The compound, which is an intermediate, in or at the beginning of a secondary metabolite biosynthetic route, stands a good chance of increasing the yield of the final product. Attempts to induce or increase the production of plant secondary metabolites by supplying precursor or intermediate compounds, have been effective in many cases (Anitha and Ranjitha, 2006).

Elicitors are signals triggering the formation of secondary metabolites. Use of elicitors of plant defense mechanisms, i.e. elicitation, has been one of the most effective strategies for improving the productivity of bioactive secondary metabolites. Biotic and abiotic elicitors based on their origin are used to stimulate number of secondary metabolite formation in plant cell cultures, thereby reducing the process time to get higher yield of secondary metabolites (Namdeo et al., 2002; Sharma et al., 2015). Production of some of valuable secondary metabolites using various elicitors was reported (Namdeo et al., 2002; Sharma et al., 2015; Harisaranraj et al., 2009).

\section{Steroidal Lactones Metabolism in Withania somnifera in vitro}

Withania somnifera is an important Indian medicinal plant has received considerable attention due to the potent biological properties attributed to the presence has emerged as one of the important Indian medicinal plants due to its potent biological properties. An efficient protocol was established for its regeneration and mass propagation through plant growth regulator mediated organogenesis producing up to 1368 plantlets per explant cultured in a time frame of 13 weeks. Withanolide contents (Withanone, Withaferin A, Withanolide A and Withanolide B) were analyzed in plant parts of W. somnifera and tissue cultured lines 
grown on $\mathrm{MS} / \mathrm{B}_{5}$ medium containing various plant growth regulators. Withanolides were identified by HPLC-UV (DAD) - Positive ion electrospray ionization spectroscopy. Callus cultures grown on $\mathrm{B}_{5}$ medium containing 2.0 $\mathrm{mgl}^{-1} \mathrm{NAA}$ yielded $17-30.8 \%$ Withanolides producing only Withanolide $\mathrm{A}$ and Withanone. The calli turned organogeneic when placed on MS medium amended with2.0 mgl ${ }^{-1}$ BAP in combination with1.0 $\mathrm{mgl}^{-1}$ IBA also showed the presence of Withanolide B. MS medium supplemented with $1.0 \mathrm{mgl}^{-1}$ BAP supported the multiplication of shoots and yielded significantly higher levels of all Withanolides. Chemical constituents of the plant comprise of steroidal lactones (withanolides). Modulation of Withanolides metabolism was closely observed using different PGRs mediated organogenesis (Sharada et al., 2007; 2008). Glycowithanolides have also been reported from tissue cultures of Withania somnifera (Ahuja et al., 2009).

\section{Bacoside metabolism in Bacopamonnieri (L.)Wettst in vitro}

Bacosides have received considerable attention as potent bioactive molecules due to their potent biological activities. Various studies carried out so far, most of them pertains to in vitro regeneration of $B$. monnieri plantlets, however, and none of these reports have described potential of these cultures or regenerated plants for bacoside formation. As such several reports addressed Bacoside metabolism in vitro in $B$. monnieri. The clonal propagation of $B$. monnieri through shoot tips and auxillary buds described here provided a strategy to clonally propagate plants and have more homogenous bacoside content and maintain genetic integrity of elite clone. Multiple shoot forming capability retained on long term basis. Bacoside analysis of clonally propagated plants was carried out by means of
HPLC and LC-MS showed Bacoside A3 and A2 as major bacosides. Their structure and preferred confirmation were determined on the basis of spectroscopic data. The total bacosides content was comparable and essentially the same as detected for mother plant. The total bacosides ranged between 2.30 to $2.70 \%$ on dry weight basis. The foliage collected from field grown clonally propagated plants and naturally grown plants at 2 stages of development; vegetative and reproductive stages, were harvested and dried at $50 \pm 2^{\circ} \mathrm{C}$ overnight. The dried samples $(10 \mathrm{~g}$ each) of powdered plant material were soxhlet extracted with methanol $(150 \mathrm{ml})$ for $4 \mathrm{~h}$ at room temperature. The extract was concentrated to $60 \mathrm{ml}$ under vacuo in water $(90 \mathrm{ml})$ and successively extracted with nhexane (100 $\mathrm{ml} \mathrm{x} \mathrm{3)} \mathrm{and} \mathrm{n-butanol} \mathrm{(50} \mathrm{ml} \mathrm{x}$ 4). Butanol extracts were dried under vacuo to obtain total bacosides. Thin layer chromatography (TLC) and HPLC were used for identification and quantification of bacosides. The presence of bacoside (A3 + A2) was additionally confirmed by LC-MS. HPLC - quantitative analysis of bacosides was performed by HPLC. Calibration curves for bacoside A2 and A3 were prepared on the basis of standard mixture. The concentrations of bacoside A2 and A3 were in the ratio of 41:9 as determined by HPLC at $210 \mathrm{~nm}$. Efficient calibration coefficients were obtained for these two standards. The values for the calibration coefficients were 0.99985 and 0.99782, respectively. Bacoside A3 and A2 eluted at retention times of 8.452 and 9.470 minutes which exhibited molecular ion peaks respectively at $\mathrm{m} / \mathrm{z} 951[\mathrm{M}+\mathrm{Na}]+$ and $921[\mathrm{M}+\mathrm{Na}]+\mathrm{in}$ the positive mode (Ahuja et al., 2005; Sharma et al., 2015).

\section{In vitro plumbazin production from cultured tissue of Plumbago zeylanica}

Plumbagin is an important bioactive molecule known for its broad range of pharmacological 
activities, such as anticancer, antimicrobial, antifertility and insecticidal. Natural occurrence of plumbagin occurs in several plant species of the family Plumbaginceae and Droseraceae. Plumbaginceae is found in Africa, many parts of Asia and Europe while Droseraceae (sundew) family is found in many temperate and tropical regions of the world. Roots of Plumbago species are the main source of plumbagin production. Plumbago zeylanica L., belongs to family Plumbaginaceae, is a rambling subscandent perennial herb or under shrub. The roots of Plumbago zeylanica $\mathrm{L}$ are used extensively in China and other Asian countries for the treatment of cancer, rheumatoid arthritis, dysmenorrheal and contusion of extremities. The root stimulates the secretion of sweat, urine and bile and has a stimulant action on the nervous system. Extract of the root is given internally or applied to the sodium uteri causes abortion.

Production of plumbagin by plant cell cultures is receiving more attention because native plants such as Plumbago sp. and Drosophyllum $s p$. produce only small amounts of this compound after 2-6 years of growth (Kitanov and Pashankov, 1994). Production of plumbagin from $P$. rosea cell cultures have been reported (Komaraiah et al., 2001). But these cultures produced plumbagin in very small amount and not found suitable for commercial exploitation. Plant cell cultures could be a potential source of a wide variety of valuable pharmaceuticals, however, only a few commercial processes based on plant cell cultures exist at the moment. The main drawback of cultured plant cells is lower yields, stability of the cell lines, inconsistency in the production and the storage of the metabolites within the cells or vacuoles. Recovery of products from cultures needs harvesting and extraction of the cell suspension. Cell suspension culture may be used for whole or partial synthesis of secondary plant products. Although a few studies have been conducted in some laboratories of worldwide to produce secondary metabolites in Plumbago zeylanica but reports are not encouraging. Experiments were conducted to quantify secondary metabolite production in calli obtained from nodal segment and leave disc cultures and cell clumps/embryoid acquired from cell suspension cultures of Plumbago zeylanica. Higher plumbazin content was detected in one-month-old friable callus $(0.428 \mathrm{mg} / 100 \mathrm{gm})$, cell clumps/embryoids $\left(0.357 \mathrm{mg} . \mathrm{l}^{-1}\right)$ as well as in two-months-old rhizogenic calli (1.257 $\mathrm{mg}$ per gm) on MS culture medium amended with $3.0 \mathrm{mgl}^{-1}$ 2,4-D in combination with $0.5 \mathrm{mgl}^{-1}$ IBA. Linearly increased plumbagin concentration in both callus and cell suspension culture filtrate was recorded with increased concentration of 2, 4D (Patidar et al., 2015).

\section{Glychyrhizin and related terpenoids}

Simultaneous qualitative and quantitative assessment of eight flavonoids and two terpenoids was performed in fourteen in vitro raised morphogenic cultures of Glycyrrhizaglabra. Our study revealed that the spectrum and production of ten compounds, under investigation, was higher in organized tissue than the undifferentiated mass, however, aerial portions of the in vitro raised plants (leaf and stem) were found to be devoid of glycyrrhizin. Additionally, an interesting correlation was revealed between glycyrrhizin accumulation and various differentiation stages of the plant. We also evaluated cytotoxic effect of the extracts against panel of human cancer cell lines in vitro, among which, pancreatic cell line (MIA-PaCa-2) was found to be sensitive to all the fourteen extracts investigated. Notably, extracts with higher glycyrrhizin content displayed cell inhibition activity of the order of $44 \%$ against breast cancer cell line. 
Overall, our findings demonstrated that the metabolite spectrum of varied in vitro raised morphogenetic lines, at different stages of maturation, might offer a powerful tool to understand the regulatory aspects of the concerned metabolite pathway and their consequent role in differentiation. Results presented here have revealed that the phytochemical profiling was found associated with the organogenesis (Gupta et al., 2013).

Recently simultaneous qualitative and quantitative assessment of eight flavonoids and two terpenoids were performed in fourteen in vitro raised morphogenic cultures of Glycyrrhiza glabra. Our study revealed that the spectrum and production of ten compounds, under investigation, were higher in organized tissue than the undifferentiated mass, however, aerial portions of the in vitro raised plants (leaf and stem) were found to be devoid of therapeutically relevant triterpenoid, glycyrrhizin. A correlation was observed between cell maturation, morphological differentiation and glycyrrhizin accumulation. Mature stolons (4 months) were characterized by the maximum accumulation of glycyrrhizin $(8.60 \mathrm{~g} / \mathrm{mg})$ in in vitro plantlets. The cytotoxic effect of the extracts evaluated against a panel of human cancer cell lines (in vitro) indicated that the pancreatic cell line (MIAPaCa-2) were sensitive to all the fourteen extracts investigated (Saima et al., 2015).

\section{Amarogentin and amaroswerin}

Chemical investigations of various in vitro developed morphotypes revealed that proliferating shoot cultures produce bioactive molecules amarogentin and amaroswerin equal to the parental plants. As the herb is directly being used by the industry without any downstream process of extraction of active principal, the shoot cultures seem to have potential for direct use in the industry. Studies are being carried out to explore possibility for an alternative supply route through biotechnological production of biomass/product using shoot cultures in a bioreactor. Present study is aimed at to develop procedure for a. development of shoot cultures of Swertia chirayita; b. culturing shoot material in tissue culture under conditions that organogenically produce a proliferating of shoot biomass; and c. standardization of the conditions for harvesting said shoots and/or leafy material while at green, actively-growing, nonsenescent stage and produce desired amount of amarogentin and amaroswerin (Sushmaet al., 2009).

\section{Reserpine and Ajmalicinemetabolism in Rauvolfia serpentina}

Rauvolfia serpentine is an erect evergreen, woody perennial shrub and commonly known as sarpagandha. Major constitutes of sarpagandha roots are reserpine, rescinnamine, deserpidine and yohimbine (Klyshnichenko et al., 1995). According to Ayurveda, the roots and whole plants are used for the treatment of cardio vascular disorder, snake bite, rheumatism, hypertension, insanity, epilepsy and hypochondria infusion, decoction and extract of the roots are employed to increase uterine contraction for expulsion of foetus, to treat painful affection of bowels, diarrhoea, dysentery, cholera and colic value of sarpagandha root depends on total alkaloid content and proportion of reserpine and ajmalcine alkaloids present in it. Reserpine has remarkable physiological activities, which have led to its extensive use in the treatment of hypertension, nervous and mental disorders. It is also used in headache and asthma. Ajmalicine has remarkable physiological activities, which have led to its extensive use as anti-hypertensive, antibacterial and sedative in drugs (Rojaet al., 1990). 
Experiments were conducted to quantify secondary metabolite production in callus and cell suspension culture of Rauvolfia serpentina. Reserpine and ajmalicine were detected in one-month-old callus as well as in cell suspension cultures. MS medium supplemented with $1.0 \mathrm{mgl}^{1}$ 2,4-D in combination with $0.5 \mathrm{mgl}^{-1}$ IBA indicates the highest recovery of reserpine content in both callus and liquid suspension medium of onemonth age. Increasing concentration of 2,4-D in liquid medium drastically decreased reserpine content. Linearly decreased ajmalicine concentration in both callus and cell suspension culture was recorded with increased concentration of 2,4-D. Embryogenic cell suspension culture of $R$. serpentine may be proved quite useful and convincing tool to improve the yield of secondary metabolites reserpine and ajmalicine in in vitro. Both alkaloids may be further produced in commercial scale by bioreactor cultivation (Uikey et al., 2014).

\section{Volatile terpenoids}

The biosynthetic capacity of in vitro proliferating shoots and regenerated callus clones has been evaluated for essential oil production. On evaluation it was found that the essential oil isolated from foliage of proliferating shoots and regenerated plantlets was a complex mixture with 49 components, 25 of which were identified, corresponding to $80 \%$ of the total oil content. The analysis of the identified constituents included monoterpene hydrocarbon (43\%), oxygenated monoterpene $(31 \%), \quad$ sesquiterpene hydrocarbons $(7.4 \%)$ and oxygenated sesquiterpenes $(4.0 \%)$. The major constituents were myrcene, limonene, (E)-linalool, (Z)ocimene and caryophyllene oxide (Ahuja et al., 2005).Recently reported study revealed comparative similarity of volatile constituents of naturally grown and micropropagated plants (Ahuja et al., 2016).
The production of chemicals and pharmaceuticals using plant cell cultures has made great strides building on advances in plant science. The use of genetic and rDNA technology tools and regulation of pathways for secondary metabolism have provided the basis for the production of commercially acceptable levels of products. However, despite progress strategies are still needed to develop an information based on a cellular and molecular level for the most of the molecules. Because of the complex and incompletely understood nature of plant cells in in vitro cultures, case-by-case studies have been used to explain the problems occurring in the production of secondary metabolites from cultured plant cells. As such focused approach depending upon nature of compound and resource plant and culture type needs to be taken into consideration for successful application of tissue culture to harvest appreciable level of compound for production at commercial level. Knowledge concerning pathway dissection at molecular level is required to be developed for each compound to harvest the benefit of system biology and metabolic approaches for production at commercial level.

\section{References}

Ahuja, A. (1989). Useful bioactive products from plant tissue cultures. $C E W, \mathrm{XXIV}$ 46-48.

Ahuja, A., Gupta K. K., Khajuria, R.K., Sharma, A., Kumar, A., Sharada, M. and Kaul, M.K. (2005).Plant Biotechnology and its Applications in Tissue Culture Vol I; Ashwini Kumar, Shikha Roy (Eds.) Chapter 17.

Ahuja, A., Bakshi, S.K., Sharma, S.K., Thappa, R. K., Agarwal, S.G., Kitchlu, S., Paul, R. and Kaul, M.K. (2005). Production of volatile terpenes by proliferating shoots and micropropagated plants of Santolinacha 
maecyparissus L. (Cotton Lavender) Flavour Frag. J; 20:463.

Ahuja, A., Kaur, D., Sharada, M., Kumar, A. Krishan, A.S. and Dutt, P.(2009). Glycowithanolide accumulation in vitro shoot cultures of Indian Genseng (Withania somnifera Dunal) Nat. Prod. Comm. 4 (4): 479-482.

Ahuja,A.,Kitchlu, S., Bakshi, S.K., Tripathi, M.K. and Tiwari G.(2016). Volatile terpenoid spectrum of essential oil of micropropagated and naturally grown plants in cotton lavender (Santolina chamaecyparissus L.). International Journal of Agriculture Sciences, 8: 53, 2718-2727.

Anitha, S. and Ranjitha K.B.D. (2006). Stimulation of reserpine biosynthesis in the callus of Rauvolfia tetraphylla L. by precursor feeding. Afr. J. Biotecnol. 5 (8): 659-661.

Balandrin, M.F. and Klocke, J.A. (1988). Medicinal, aromatic and industrial materials from plants. In Biotechnology in Agriculture and Forestry. Bajaj, YPS (Ed.). Vol. 40, Springer Verlag, Berlin. pp 1-35.

Dornenburg, H. and Knorr, D. (1995). Strategies for the improvement of secondary metabolite production in plant cell cultures. EnzMicrob Tech. 17: 674-684.

Harisaranraj, R., Suresh, K., and Babu, S. (2009). Production of reserpine in somatic embryos of Rauvolfia serpentina cultured in bioreactors by the induction of elicitor (methyl jasmonate). Global J. Biotech. \&Biochem. 4(2):143147.

Gupta, S., Pankaj, P., Ajai, P., Gupta, M..K., Verma, A., Ahuja, A., Vishwakarma, R.A. (2013). Direct rhizogenesis, in vitro stolon proliferation and high throughput regeneration of plantlets in Glycyrrhiza glabra. Acta Physiologiae Plantarum 09/2013; 35(9): 2669-270.
Namdeo, G. S., Patil, D. and Fulzele, P. (2002). Influence of fungal elicitors on production of ajmalicine by cell cultures of Catharanthus roseus. Biotechnol. Prog. 18: 159-16.

Oksman-Caldentey, K.M. and Inze, D. (2004). Plant cell factories in the postgenomic era: new ways to produce designer secondary metabolites. Trends Plant Sci. Pp. 99.

Patidar, S. L., Tiwari, G., Tripathi, M. K., Patel, R. P. and Mishra S.N. (2015). In vitro biosynthesis and quantification of plumbazin in cell suspension culture of Plumbagozeylanica. Medicinal Plants International Journal of Phytomedicines and Related Industries. 7 (1): 60-67.

Ramawat, K.G., Sharma, R. and Suri, S.S. (1999). Medicinal Plants In: Biotechnology- Secondary metabolites (Ed. by Ramawat, K.G. and Merillon, J.M.), Oxford and IBH, India. pp: 66367.

Rao, S.R. and Ravishankar, G. A. (2002). Plant cell cultures: chemical factories of secondary metabolites. Biotechnol. Adv. 20: 101-153.

Roja, G., Benjamin, B.D., Heble, M.R., Patankar, A.V. and Sipahimalani, A. T. (1990). The effect of plant growth regulators and nutrient conditions on growth and alkaloid production in multiple shoot cultures of Rauvolfia serpentina. Phytotherapy Res. 4(2): 4952.

Khan, S., Pandotra, P., Malik, M.M., Kushwaha, M., Sharma, R., Jain, S., Ahuja, A. Amancha, V., Bhushan, S., Guru, S. K., Gupta, A. P., Vishwakarma, R. and Gupta S. (2015). Terpenoid and flavonoid spectrum of in vitro cultures of Glycyrrhiza glabra revealed high chemical heterogeneity: platform to understand biosynthesis. Plant Cell Tiss. \& Org.Cult.11/2015; 
124(3). DOI: 10.1007/s11240-0150910-4.

Sharma, M., Rajinder, G., Khajuria, R. K. Sharada, M. and Ahuja, A.(2015). Bacoside biosynthesis during in vitro shoot multiplication in Bacopamonnieri (L.) Wettst. grown in Growtek and air lift bioreactor. Indian J. Biotechnol. 11/2015; 14(4).

Sharma, M., Ahuja, A., Gupta, R. and Sharada, M.(2014). Enhanced bacoside production in shoot cultures of Bacopamonnieri under the influence of abiotic elicitors. Natural Product Research, 12/2014; 29(8). DOI:10.1080/14786419.2014.986657.

Sharada, M.,Ahuja, A., Suri, K.A., Vij, S. P., Khajuria, R.K., Verma, V. and Kumar, A. (2007). Withanolideproduction by in vitro cultures of Withania somnifera (L) Dunal and its association with differentiation, Biologia Plantarum; 51:161-164.

Sharada, M., Ahuja, A and Vij, S.P. (2008). Recent Advances in Plant Biotechnology (Eds. Ashwini Kumar and SK Sopory) I.K. International Pvt. Ltd., New Delhi, India. Chapter 41 pp 645-667.

Koul, S., Suri, K., Dutt, A. P. Sambyal,M., Ahuja, A. and Kaul, M.K. (2009).Methods in Molecular Biology, Protocols for in vitro cultures and secondary metabolite analysis of aromatic and medicinal plants, Vol. 547S. Mohan Jain and Praveen K. Saxena (eds.), Humana Press, a part of Springer Science + Business Media, LLC Chapter 12 pp 140-153.

Uikey, D.S., Tiwari, G., Tripathi, M.K. and Patel, R.P. (2014). Secondary metabolite production of reserpine and ajmalicine in Rauvolfia serpentina (L.) Benth. through callus and cell suspension culture. International Journal of Indigenous Medicinal Plants, 47: 1633-1646.

Verpoorte, R. and Memelink, J. (2002). Engineering secondary metabolite production in plants. Curr. Opin. Biotechnol. 13: 181-187.

\section{How to cite this article:}

Tripathi, M.K., Nishi Mishra, Sushma Tiwari, Chitralekha Shyam, Sonali Singh and Ashok Ahuja. 2019. Plant Tissue Culture Technology: Sustainable Option for Mining High Value Pharmaceutical Compounds. Int.J.Curr.Microbiol.App.Sci. 8(02): 1002-1010. doi: https://doi.org/10.20546/ijcmas.2019.802.116 